\title{
Overexpression of hTERT in potentially malignant colorectal laterally spreading tumors
}

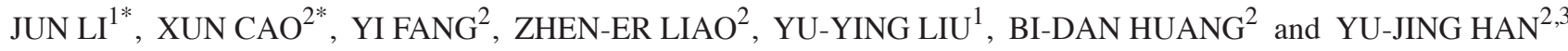 \\ ${ }^{1}$ Department of Traditional Chinese Medicine, Nanfang Hospital, Southern Medical University, Guangzhou 510515; \\ ${ }^{2}$ State Key Laboratory of Oncology in South China; ${ }^{3}$ Department of Preclinical Medicines, \\ Cancer Center, Sun Yat-Sen University, Guangzhou 510060, P.R. China
}

Received November 15, 2012; Accepted February 27, 2013

DOI: $10.3892 / \mathrm{mmr} .2013 .1376$

\begin{abstract}
Human telomerase reverse transcriptase (hTERT) is the main subunit of the core enzyme telomerase, which consists of three subunits. Telomeres are essential for chromosomal stability and integrity, protecting the ends of chromosomes from degradation and preventing chromosomal end fusions and recombination. A loss of telomere function is a major mechanism for the generation of chromosomal abnormalities. Telomere shortening leads to mutations, chromosome rearrangements and translocations. Colorectal laterally spreading tumors (LSTs) are a special type of superficial colorectal tumor. They are considered to have a high malignancy potential. The aim of the present study was to characterize the expression of hTERT in an LST cell line and paraffin sections. Immunohistochemistry was utilized to examine the protein expression of hTERT in the LST cell line, 48 resected LSTs, 48 protruded-type colorectal adenomas (PAs) and 48 normal mucosa samples. Statistical analyses were applied to test the associations between hTERT expression and clinicopathological parameters. The present study demonstrated that the positive expression levels of hTERT in LSTs, PAs and normal mucosa were $60.4,22.9$ and $10 \%$, respectively. Compared with polypi and normal mucosa, the expression levels of hTERT were significantly increased in LSTs. The expression of hTERT was also observed in the LST cell line. The expression of hTERT was significantly higher in LSTs, which may indicate a potential for malignancy.
\end{abstract}

Correspondence to: Dr Yu-Jing Han, Department of Preclinical Medicines, State Key Laboratory of Oncology in South China, Cancer Center, Sun Yat-Sen University, 651 Dongfeng Road East, Guangzhou, Guangdong 510060, P.R. China

E-mail: hanyj@sysucc.org.cn

*Contributed equally

Key words: human telomerase reverse transcriptase, laterally spreading tumors, immunohistochemistry, potential significance

\section{Introduction}

Colorectal laterally spreading tumors (LSTs) of the colon and rectum are defined as lesions $>10 \mathrm{~mm}$ in diameter with a low vertical axis that extend laterally along the luminal wall (1). The clinical characteristics of LSTs include a flat and superficial growth pattern. Although LSTs are known as adenomas or 'pre-existing cancers', they have been hypothesized to have malignant potential. LSTs are divided into two macroscopic subtypes, flat (F)-type and protruded-type (2). Despite the distinctive biological behaviors of LSTs, a number of genetic alterations have been reported, including $K$-ras and $p 53$ mutations and cyclooxygenase 2 overexpression (3-6). Wang et al (7) observed that the expression of E-cadherin, $\beta$-catenin, glycogen synthase kinase-3 $\beta$ (GSK-3 $\beta$ ), cyclin D1 and c-myc was positive in LSTs. Human telomerase reverse transcriptase (hTERT/Hest2/TP2), the main subunit involved in telomerase reverse transcription, is considered as one of the most important proteins affecting telomerase activity $(8,9)$. However, hTERT expression in LSTs has not been thoroughly studied. Thus, this study aimed to elucidate the expression levels of hTERT in LSTs, colorectal tumors with a high malignancy potential.

\section{Materials and methods}

LST cell line. The novel LST cell line was obtained from the Department of Gastroenterology, Nan Fang Hospital, Affiliated Hospital of Nan Fang Medical University, China. The LST cell line was established from a human rectal villous adenoma obtained using endoscopic resection. A magnifying endoscope showed a flat granular tumor with nodes $(70 \times 60 \mathrm{~mm}$ ) in the rectum (Fig. 1A). Pathological examination of the biopsy specimen revealed that it had the characteristic morphology of a villous adenoma accompanied by moderately severe atypical hyperplasia (Fig. 1B). The LST cell line was grown in RPMI-1640 medium (Invitrogen, Carlsbad, CA, USA) containing $100 \mathrm{~mol} / \mathrm{l}$ fetal calf serum and antibiotics. Cells were cultured in a humidified $5 \% \mathrm{CO}_{2}$ atmosphere. Cells from the primary passage of the LST were cultured for $48 \mathrm{~h}$ and fixed in ice-cold pure alcohol at $-20^{\circ} \mathrm{C}$ (Fig. 1C).

Tissue specimens. A total of 48 LSTs were collected by endoscopic resection at the Cancer Center of Sun Yat-Sen 
University between August 2007 and July 2010. The histology of the tissue specimens revealed that they were all adenomas. In total, 48 protruded-type colorectal adenomas (PAs) and 48 normal mucosa samples were used as negative controls. The study was performed in accordance with institutional ethical guidelines and was approved by the Scientific Committee of Cancer Center of Sun Yat-Sen University. Informed consent was obtained from all patients.

Immunohistochemistry. Cells were grown on chamber slides and stained for hTERT expression with a specific antibody (Beijing Zhongshan Biology Technology Ltd., Beijing, China). The specimens were fixed in 10\% formalin solution and then embedded in paraffin. Serial sections were cut and prepared for hematoxylin and eosin (H\&E) staining and immunohistochemistry, then rehydrated through graded alcohol. Antigen retrieval was performed by microwaving at moderate power for $3 \mathrm{~min}$, followed by high power for $10 \mathrm{~min}$ in $0.01 \mathrm{M}$ citrate buffer (PH 6.0). Endogenous peroxide activity was quenched by incubation with $3 \%$ hydrogen peroxide in methanol for $20 \mathrm{~min}$. The slide was then incubated with a mouse monoclonal whole-serum antibody against hTERT (1:25 dilution; NCL-hTERT; Novo Castra, Newcastle upon Tyne, UK) at room temperature for $2 \mathrm{~h}$. HRP-conjugated secondary antibody (anti-mouse, EnVision System kit; Dako, Carpinteria, CA, USA) was then applied for $60 \mathrm{~min}$. Slides were rinsed in TBST twice. 3,3'-Diaminobenzidine was used as the chromogen. Slides were counterstained with hematoxylin, dehydrated in a graded series of ethanol, mounted on slides and cover slipped (10). For negative controls, normal mouse serum was added instead of the primary antibody. Epithelium and glands with a normal appearance in each section provided positive internal controls for binding of the primary antibodies.

Positive expression of hTERT in epithelial tissue cells revealed a primarily cytoplasmic or nuclear pattern with yellow or brown staining. All sections were blindly and independently assessed microscopically by two trained pathologists. The intensity and distribution of immunohistochemical staining for hTERT were evaluated (7). The intensity of staining was assessed with a semi-quantitative scoring system as follows: 0 , negative staining; 1 , weak staining; 2 , moderate staining; and 3 , strong staining. The distribution of staining was graded by the percentage of stained cells in the region of interest as follows: $0,<10 \%$ of tumor cells were positive; $1,10-50 \%$ of tumor cells were positive; $2,50-75 \%$ of tumor cells were positive; and 3,>75\% of tumor cells were positive. An overall score was obtained by combining the intensity and distribution of positive staining. Cases with 0 points were considered to be negative (0), cases with a final score of 1-3 as weakly positive (1+), cases with a final score of 4-7 as moderately positive (2+) and cases with a final score of $>7$ as strongly positive (3+).

Statistical analysis. Student's t-test was used to compare age, gender and tumor size features between the PAs and LSTs. A Chi-square test was used for comparing the remaining parameters between these two groups. To evaluate differences in the expression of hTERT proteins among LSTs, PAs and normal mucosa, the Kruskal-Wallis test was used, and further analysis with a Bonferroni test was used to compare any two
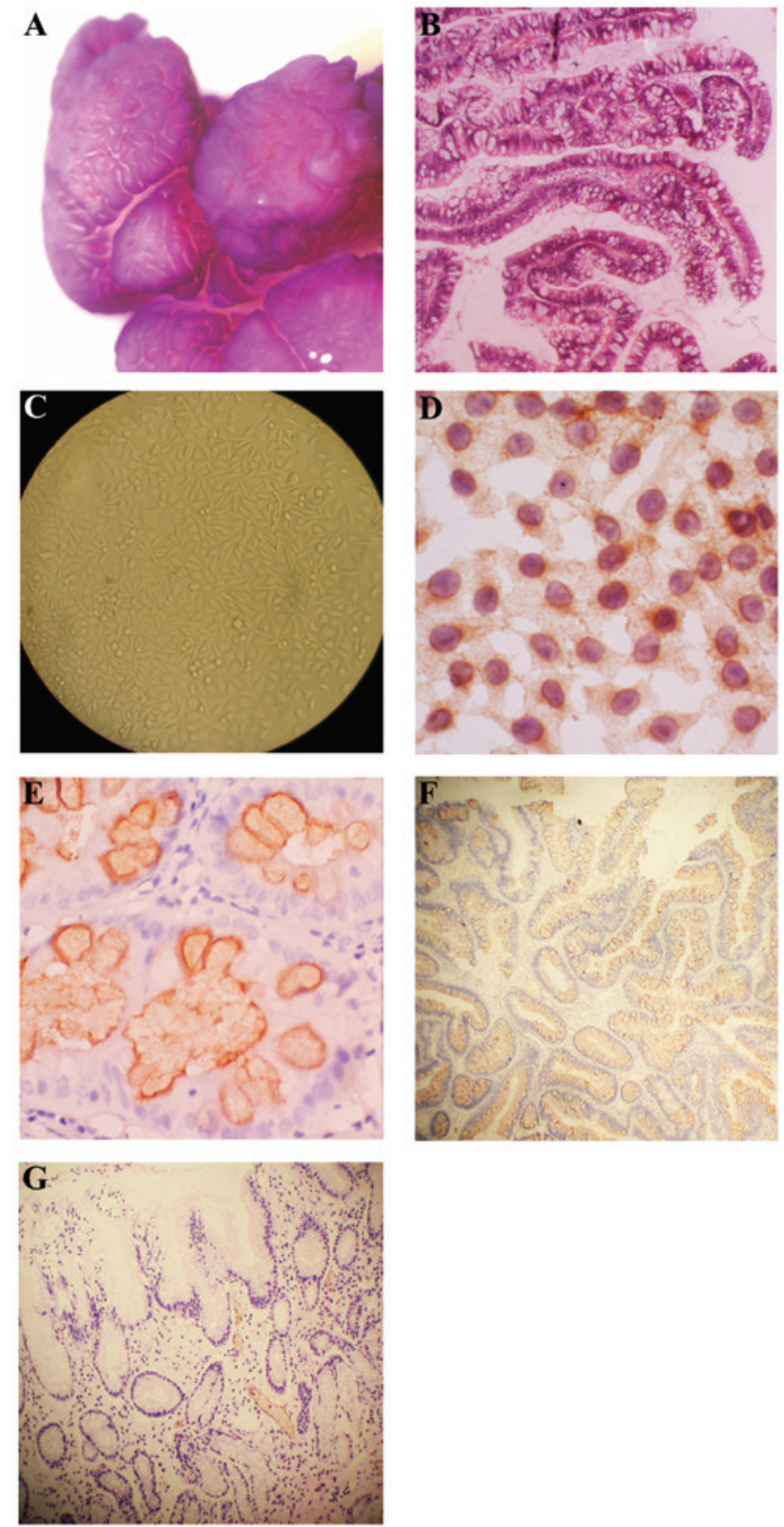

Figure 1. (A) Tissue obtained by endoscopic resection; (B) histopathology of the colorectal laterally spreading tumor (LST) tissue (x100): A villous adenoma accompanied by moderately severe atypical hyperplasia; $(\mathrm{C})$ the primary cultured LST cell line (x100); (D) immunohistochemistry of the cell line (x400); (E) LST (villous adenoma) hTERT expression (x100); (F) LST (tubular adenoma) hTERT expression (x250); (G) the negative control (x100). hTERT, human telomerase reverse transcriptase.

groups. All statistical tests were two-sided and $\mathrm{P}<0.05$ was considered to indicate a statistically significant difference. The statistical analyses were performed using the SPSS 13.0 software package (SPSS, Chicago, IL, USA).

\section{Results}

Patient characteristics. The clinicopathological features of LSTs and PAs are summarized in Table I. The mean age of patients with PAs was 62.3 years, whereas the mean age of those with LSTs was 64.1 years. No significant difference was identified in age $(\mathrm{P}=0.163)$, gender $(\mathrm{P}=0.142)$, histology 
Table I. Patient characteristics.

\begin{tabular}{lccr}
\hline Characteristics & PAs & LSTs & P-value \\
\hline Age (years) & & & 0.163 \\
Mean \pm SD & $62.3 \pm 11$ & $64.1 \pm 11.8$ & \\
Gender & & & 0.142 \\
Male/female & $36 / 12$ & $28 / 20$ & \\
Tumor size (mm) & & 0.032 & \\
Mean \pm SD & $15.5 \pm 6.1$ & $28.2 \pm 16.3$ & \\
Location, n (\%) & & & $<0.001$ \\
Proximal colon & $6(12.5)$ & $20(41.7)$ & $<0.001$ \\
Distal colon & $30(62.5)$ & $6(12.5)$ & $<0.001$ \\
Rectum & $12(25.0)$ & $22(45.8)$ & 0.106 \\
Histology, n (\%) & & & 0.137 \\
Tubular & $18(37.5)$ & $12(25)$ & \\
Tubulovillous & $26(54.2)$ & $34(70.8)$ & \\
Villotubular & $4(8.3)$ & $2(4.2)$ & \\
Grade, n (\%) & & & \\
Low & $28(58.3)$ & $20(41.7)$ & \\
High & $20(41.7)$ & $28(58.3)$ & \\
\hline
\end{tabular}

PAs, protruded-type colorectal adenomas; LSTs, colorectal laterally

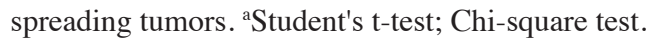

$(\mathrm{P}=0.137)$ or grade of intraepithelial neoplasia $(\mathrm{P}=0.150)$ between the LSTs and PAs. With regard to the tumor size, the mean size of PAs was $15.5 \mathrm{~mm}$, whereas the mean size of LSTs was $28.2 \mathrm{~mm}$ and a significant difference was identified $(\mathrm{P}=0.032)$. In total, $43.5 \%$ of LSTs were located in the proximal colon; however, only $12.5 \%$ of PAs were located in the proximal colon. Additionally, PAs were identified more frequently in the distal colon $(62.5 \%)$, whereas only $(8.7 \%)$ of LSTs were located in the distal colon $(\mathrm{P}<0.001)$.

Immunohistochemical staining in the LST cell line. As shown in Fig. 1D, the positive expression of hTERT was localized to the cytoplasm or nuclei in LST cultured cells.

Immunohistochemical expression in parafin sections. The expression of hTERT localized to the cytoplasm among PAs, LSTs and normal mucosa is shown in Fig. 1E-G. The expression of hTERT was shown to be significantly different among the three groups $(\mathrm{P}<0.001)$. The positive expression levels of hTERT in the LSTs, PAs and normal mucosa were 60.4, 22.9 and $10 \%$, respectively. The weak nuclear expression of hTERT was demonstrated as $18.8 \%$ in LSTs (9/48) and $16.7 \%$ in PAs (8/48). Further analysis with the Bonferroni test revealed that differences in the expression of hTERT occurred between LSTs, normal mucosa samples $(\mathrm{P}<0.001)$ and PAs $(\mathrm{P}<0.001)$, but not between PAs and normal mucosa $(\mathrm{P}=0.201$; Table II).

\section{Discussion}

Since the discovery of the telomerase enzyme in humans, it has become one of the most notable tumor markers and its
Table II. Expression of hTERT localized to the cytoplasm in PAs and LSTs.

\begin{tabular}{lrrrrrr}
\hline & & \multicolumn{5}{c}{ hTERT $^{\text {a }}$} \\
\cline { 3 - 5 } Category & No. & 0 & $1+$ & $2+$ & $3+$ & P value $^{\text {b }}$ \\
\hline LSTs & 48 & 19 & 9 & 14 & 6 & $<0.001$ \\
PAs & 48 & 37 & 8 & 2 & 1 & $<0.001$ \\
Normal mucosa & 20 & 18 & 2 & 0 & 0 & 0.021 \\
\hline
\end{tabular}

LST, colorectal laterally spreading tumors; PAs, protruded-type colorectal adenomas. ${ }^{\text {a?; }}{ }^{\mathrm{b} B}$ Bonferroni testing was used to compare two groups between LSTs, PAs and normal mucosa. The corrected $\alpha=0.05 / 3=0.017$.

significance in diagnostic, prognostic and therapeutic applications has been revealed. Extensive investigation has identified three core components of the enzyme, of which the catalytic subunit hTERT appears to be the most significant. More than $85 \%$ of tumors express telomerase, which provides an effective tool for the diagnosis, prognosis and treatment of malignancy. Telomerase activity is readily detected in $\sim 3000$ human malignant tumors, including breast, prostate, lung, liver, pancreatic and colon cancer. Telomerase activity is absent in differentiated somatic tissues (11-20); however, in the majority of tumors, overexpression of hTERT is accompanied by an increase in telomerase activity. The presence of hTERT in tissue samples and cells lines may be beneficial for identifying the stage and grade of the tumor. Depending on the type of test sample (fixed tissue, whole cells or tissue lysates), hTERT may also be detected by fluorescence in situ hybridization (FISH) (21). In a number of cases, chronic conditions of specific tissues (e.g., liver cirrhosis) exhibit weak telomerase activity (22). In certain tissues, two types of hTERT distribution patterns are observed, diffused and focal (21). In others, a perinuclear and/or cytoplasmic staining pattern is observed (22). The hTERT-positive cells with diffuse expression tend to have a higher proliferative index and the tumors are smaller in size compared with the focally distributed cells. hTERT-positive cells with focal distribution have a reduced survival time. The type of hTERT distribution in certain cases correlates with the clinicopathological parameters, while in others there is no association between hTERT expression and the proliferative index, grade or stage of the tumor.

To estimate the changes in hTERT expression between LSTs and PAs, we examined the expression of hTERT using immunohistochemical staining of the cell line and tumor tissues. The hTERT protein is mainly expressed in the cytoplasm of cells, with a lower level of expression in the nucleus. The accumulation of hTERT within the cytoplasm and nucleus is associated with colorectal cancer, in addition to other types of cancer. We observed that the cytoplasmic expression of hTERT was significantly higher in LSTs than in PAs (60.4 vs. 22.9\%). Our data demonstrated that LSTs, as a type of precancerous lesion, may have the potential for malignancy. Kawanishi-Tabata et al (23) examined 122 colorectal carcinomas, including 52 cases of colon cancer and 70 cases of rectum cancer, and $80 \%$ of these carcinomas were positive for telomerase activity. Wang 
et al (7) reported that the expression of hTERT in colorectal carcinomas was $77.8 \%$. Ohnishi et al (10) showed that the immunohistochemical expression of hTERT in the peripheral vein of colorectal cancer was $81 \%$. However, in our study, the expression levels of hTERT in LSTs and PAs were lower than in the above-mentioned studies. By contrast, it has been reported that the expression of hTERT or telomerase activity does not always correlate with cancer progression in colon carcinoma, or even in bladder carcinoma (23). Additionally, telomerase activity has been shown to be regulated by hormones, growth factors and inflammatory mediators (24-26). In this study, the expression of hTERT was localized to the cytoplasm in LST cultured cells and hTERT immunoreactivity was positive. We may explain this result by speculating that the LST cell line was already in the malignant transformation process to a certain extent. It has been reported that tumor cell lines Lan-1, HeLa and Co115 are telomerase-positive (27). A study describing the establishment of the LST cell line demonstrated that the histopathology of a nude mouse xenograft tumor was a poorly differentiated adenocarcinoma and cells cultured from the xenograft tumor had the same properties as the original cell line (28). Thus, the LST cell line has higher malignancy than normal adenomas. To the best of our knowledge, this is the first study estimating telomerase activity in LSTs, including hTERT immunoreactivity in tissue samples and LST cultured cells.

In conclusion, the present study showed that activation of the hTERT protein appears to be higher in LSTs than in PAs and normal mucosa samples. The immunoreactivity expression of hTERT in the LST cultured cells was positive. It may revealed that there are some correlation between malignant tendency and LST cells. Although hTERT subunit expression appears to be a useful indicator for the prognosis of LST disease, the biological behaviors involved remain to be clarified.

\section{Acknowledgements}

This study was supported by the Natural Science Fund of Guangdong Province in China (No. S2011010002941). The authors thank Professor Bo Jiang and Professor Hong-Quan Zhang for their suggestions for this study.

\section{References}

1. Uraoka T, Saito Y, Matsuda T, et al: Endoscopic indications for endoscopic mucosal resection of laterally spreading tumours in the colorectum. Gut 55: 1592-1597, 2006.

2. Takahashi T, Nosho K, Yamamoto H, et al: Flat-type colorectal advanced adenomas (laterally spreading tumors) have different genetic and epigenetic alterations from protruded-type advanced adenomas. Mod Pathol 20: 139-147, 2007.

3. Hiraoka S, Kato J, Tatsukawa M, et al: Laterally spreading type of colorectal adenoma exhibits a unique methylation phenotype and K-ras mutations. Gastroenterology 131: 379-389, 2006.

4. Mukawa K, Fujii S, Takeda J, et al: Analysis of K-ras mutations and expression of cyclooxygenase- 2 and gastrin protein in laterally spreading tumors. J Gastroenterol Hepatol 20: 1584-1590, 2005.
5. Noro A, Sugai T, Habano W and Nakamura S: Analysis of Ki-ras and p53 gene mutations in laterally spreading tumors of the colorectum. Pathol Int 53: 828-836, 2003.

6. Kusaka T, Fukui H, Sano Y, Ueda Y, Chiba T and Fujimori T: Analysis of K-ras codon 12 mutations and p53 overexpression in colorectal nodule-aggregating tumors. J Gastroenterol Hepatol 15: 1151-1157, 2000.

7. Wang J, Wang X, Gong W, Mi B, Liu S and Jiang B: Increased expression of beta-catenin, phosphorylated glycogen synthase kinase 3beta, cyclin D1, and c-myc in laterally spreading colorectal tumors. J Histochem Cytochem 57: 363-371, 2009.

8. Frías C, Morán A, de Juan C, et al: Telomere function in colorectal cancer. World J Gastrointest Oncol 1: 3-11, 2009.

9. Kim NW, Piatyszek MA, Prowse KR, et al: Specific association of human telomerase activity with immortal cells and cancer. Science 266: 2011-2015, 1994.

10. Ohnishi T, Watanabe T, Nozawa H, Kitayama J and Nagawa H: Telomerase activity of blood samples and recurrence of colorectal cancer. Hepatogastroenterology 55: 1513-1518, 2008.

11. Tomás-Loba A, Flores I, Fernández-Marcos PJ, et al: Telomerase reverse transcriptase delays aging in cancer-resistant mice. Cell 135: 609-622, 2008.

12. González-Suárez E, Geserick C, Flores JM and Blasco MA: Antagonistic effects of telomerase on cancer and aging in K5-mTert transgenic mice. Oncogene 24: 2256-2270, 2005.

13. Shay JW and Wright WE: Telomeres and telomerase: implications for cancer and aging. Radiat Res 155: 188-193, 2001.

14. Rudolph KL, Chang S, Lee HW, et al: Longevity, stress response, and cancer in aging telomerase-deficient mice. Cell 96: 701-712, 1999.

15. Matthews P and Jones CJ: Clinical implications of telomerase detection. Histopathology 38: 485-498, 2001.

16. Yoshida K, Sugino T, Goodison S, et al: Detection of telomerase activity in exfoliated cancer cells in colonic luminal washings and its related clinical implications. Br J Cancer 75: 548-553, 1997.

17. Chatziantoniou VD: Telomerase: biological function and potential role in cancer management. Pathol Oncol Res 7 : 161-170, 2001.

18. Stewart SA and Bertuch AA: The role of telomeres and telomerase in cancer research. Cancer Res 70: 7365-7371, 2010.

19. Hahn WC: Role of telomeres and telomerase in the pathogenesis of human cancer. J Clin Oncol 21: 2034-2043, 2003.

20. Goldman MA: The role of telomeres and telomerase in cancer. Drug Discov Today 8: 294-296, 2003.

21. Falchetti ML, Pallini R, D'Ambrosio E, et al: In situ detection of telomerase catalytic subunit mRNA in glioblastoma multiforme. Int J Cancer 88: 895-901, 2000.

22. Harada K, Yasoshima M, Ozaki S, Sanzen T and Nakanuma Y: PCR and in situ hybridization studies of telomerase subunits in human non-neoplastic livers. J Pathol 193: 210-217, 2001.

23. Kawanishi-Tabata R, Lopez F, Fratantonio S, et al: Telomerase activity in stage II colorectal carcinoma. Cancer 95: 1834-1839, 2002.

24. Yang H, Kyo S, Takatura M and Sun L: Autocrine transforming growth factor beta suppresses telomerase activity and transcription of human telomerase reverse transcriptase in human cancer cells. Cell Growth Differ 12: 119-127, 2001.

25. Engelhardt M, Drullinsky P, Guillem J and Moore MA: Telomerase and telomere length in the development and progression of premalignant lesions to colorectal cancer. Clin Cancer Res 3: 1931-1941, 1997.

26. Salvia R, Bassi C, Festa L, et al: Clinical and biological behavior of pancreatic solid pseudopapillary tumors: report on 31 consecutive patients. J Surg Oncol 95: 304-310, 2007.

27. Guilleret I and Benhattar J: Demethylation of the human telomerase catalytic subunit (hTERT) gene promoter reduced hTERT expression and telomerase activity and shortened telomeres. Exp Cell Res 289: 326-334, 2003.

28. Wang XY, Lai ZS, Yeung CM, et al: Establishment and characterization of a new cell line derived from human colorectal laterally spreading tumor. World J Gastroenterol 14: 1204-1211, 2008. 